\title{
Annuntio vobis gaudium magnum - ein neues klinisches Staatsexamen!
}

\section{Martin Fey}

Prof. Dr. med., Universitätsklinik für medizinische Onkologie, Universität und Inselspital Bern

Im Anschluss findet sich eine Stellungnahme von S. Guttormsen und A.P. Perruchoud.
In ihrem Artikel loben Sissel Guttormsen und André Perruchoud [1] die Modalitäten der neuen Eidgenössischen Abschlussprüfung nach dem Medizinstudium. Währenddem Multiple-Choice-Prüfungen schon seit vielen Jahren etabliert sind, wird die neue praktische Prüfung mit trainierten Schauspielern anstelle von Patienten als besondere Verbesserung der bisherigen Praxis herausgestrichen. Früher («in der guten alten Zeit») wurden die Staatsexamens-Kandidaten mit echten Patienten konfrontiert. Sie hatten Zeit für Anamnese und klinische Untersuchung, bevor sie den Fall mit den Examinatoren im Sinne eines "practical clinical reasoning" diskutierten. Bei den neuen $\mathrm{Ob}$ jective Structured Clinical Examinations (OSCE) spielen Schauspieler eine definierte klinische Situation vor. So lernt denn ein "Appendizitis-Schauspieler», bei der Palpation seines rechten Unterbauchs durch den Prüfling situations-adäquat «auah!» zu sagen, bei der klinischen Untersuchung des linken Oberbauchs hingegen nicht. Ein gelb geschminkter Mime weist offensichtlich einen Ikterus auf, und er wird auf An-

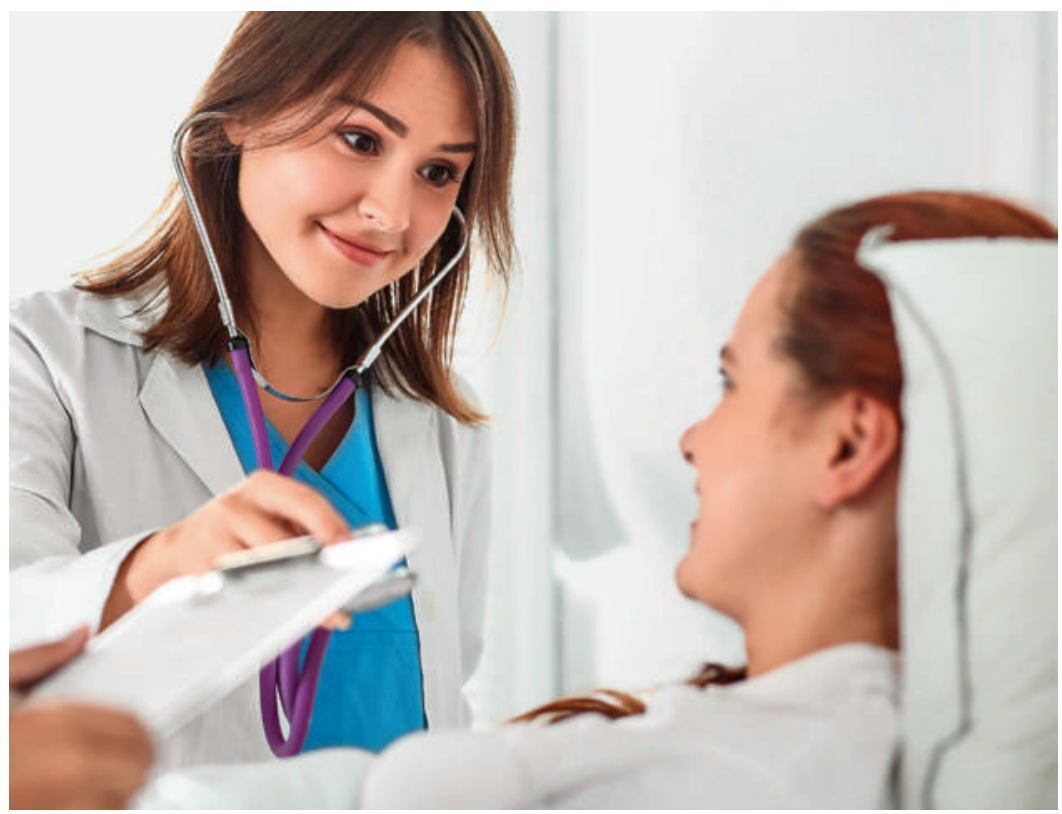

Seit 2011 untersuchen die Medizinstudenten in der Abschlussprüfung speziell geschulte Schauspieler, die eine definierte klinische Situation vorspielen. frage mitteilen, dass sein Urin dunkel sei und sein Stuhl hell. Der Kandidat muss eine Anamnese erheben, klinische Untersuchungen vornehmen, den vorgespielten Problemkreis erkennen, und Untersuchungen vorschlagen, deren Resultate ihm nur vorgelegt werden, sofern sie im Programm überhaupt vorgesehen sind. Der Examinator amtet als stummer maskenhafter Beisitzer. Er hakt im strukturierten Fragekatalog die vorge-

\section{Ein Prüfungsgespräch wäre ein Regelverstoss.}

sehenen Punkte ab, händigt Befunde aus, sofern vom Kandidaten erfragt und falls im konstruierten Fall überhaupt erhältlich. Ein Prüfungsgespräch wäre ein Regelverstoss. Clinical judgement und assoziative Reallife-Diskussionen, die Erarbeitung einer breiten Differentialdiagnose, ihre Eingrenzung auf die wahrscheinlichsten Krankheitsgruppen bis hin zur Diagnose bei einem echten Patienten, wie es für das Staatsexamen früher typisch war, sind offenbar nicht mehr gefragt. Die Prüfungskommission Humanmedizin des EDI schreibt aber die Prüfung der Kommunikationsfähigkeit und der Anwendung des Wissens des Kandidaten in der Klinik vor. Es ist schwer verständlich, weshalb dieses (vernünftige) Ziel mit Schauspielern besser erreicht werden soll als im Gespräch mit einem «richtigen" Patienten und einem interaktiven Examinator. So stellt sich überhaupt die Frage, welchen Zweck die Abschlussprüfung ("Staatsexamen») verfolgt, ob die Neuerungen überhaupt nötig waren, und wo denn ihr belegbarer Vorteil liegt. Der Artikel und das Interview tönen die Gründe nur am Rande an. Nach sechs Jahren Medizin-Studium geht es im Schlussexamen ja nicht um eine scharfe Selektion der Studenten und um eine Reduktion der Ärzteproduktion. Die Failure rate ist ohnehin minimal; das war mit den alten Prüfungsmodalitäten so, und unter den neuen Prämissen nicht anders. Die klinischen Examina dienen von jeher nicht in erster Linie der Prüfung des Fach- und Sachwissens. Das erledigen seit langem die MC-Prüfungen. Das abgeschaffte klinisch-praktische Staatsexamen animierte die Studenten, in den Prüfungsvorbereitungen Clinical reasoning zu pflegen, und ebenso vernetztes wie assoziativ-krea- 
tives Denken für die klinische Praxis zu üben; alles zweifelsohne Qualitäten, die im Arztberuf wesentlich sind. Mit MC-Fragen können diese Aspekte kaum geprüft werden, mit dem reichlich künstlichen OSCE noch weniger.

Frau Guttormsen streicht heraus, die neuen Prüfungen seien ein Erfolgsmodell, um das uns Europa offenbar beneide. Nur stellt sie leider nicht dar, wie denn der Erfolg der neuen Prüfungsform überhaupt gemessen wird, und ob prospektiv oder retrospektiv. Dies wäre genauso wesentlich wie beispielsweise die Festlegung von Endpunkten bei einer klinischen Studie.

Weshalb denn überhaupt ein Wechsel? In der früheren Art des praktischen Staatsexamens wurde zwar nicht jedem Studenten derselbe Patient zugeteilt, die Fragen wurden in der Diskussion oft spontan gestellt, der Diskurs zwischen Student und Examinator verlief (oh Schreck!) ohne zertifizierte Vorgaben und ohne intellektuelle Züchtigung durch ein Film-Skript. So total willkürlich war die alte Prüfung aber doch nicht es war Vorschrift, verschiedene Themen abzugrasen, und in der Regel waren zwei Examinatoren aus unterschiedlichen Fachrichtungen eingespannt. Während sich in der «alten» Prüfung echtes klinisches Talent und reiches Wissen des Studenten auszahlten, ist nunmehr nur noch standardisiertes Basiswissen gefragt - originelle Lösungsansätze und besondere intellektuelle Leistungen des Kandidaten ergeben keine Zusatzpunkte, da auf den Evaluationsbögen nicht vorgesehen.

Korrespondenz: Prof. Dr. med. Martin Fey Universitätsklinik für medizinische Onkologie Inselspital

CH-3010 Bern

Tel. 0316322243 martin.fey[at]insel.ch
Prüfungen, d.h. Examina genau bei der Gilde, die uns angeblich im Nacken sitzt und uns zwingt, gewachsene vernünftige klinische Prüfungsnormen über Bord zu werfen? Juristen schreiben in ihren Prüfungen freihändig seitenlange Aufsätze und «essays» zu den erteilten Themen. Die Bewertungskriterien sind im einzelnen nicht sonderlich strukturiert vorgegeben, und doch stösst sich kein Rechtsexperte an genau diesen Modalitäten, die in der Medizin nun gekippt werden, weil unsere alte Prüfungsform angeblich «juristisch» anfechtbar sei. Der Bericht von Guttormsen und Perruchoud schweigt sich darüber aus, wie mancher Rekurs unter der alten im Vergleich zur neuen Prüfungsordnung akzeptiert oder abgeschmettert wurde. Die Zahl solcher events dürfte indessen gering sein, und die Vermutung kommt hoch, dass hier in vorauseilendem Gehorsam revidiert wurde, ohne nachweislichen Handlungsbedarf.

Originelle Lösungsansätze und besondere intellektuelle Leistungen des Kandidaten ergeben keine Zusatzpunkte.

So kommt man - im Gegensatz zu den Autoren - zur nüchternen Schlussfolgerung, dass die neue Prüfung keinen sauber belegten Mehrwert schafft, wohl aber Aktivität. Ist denn der Weg das Ziel? Die Autoren drohen weitere Verfeinerungen und den Ausbau des bisherigen «Erfolgsmodells» an. Dem Häretiker grauset's. Barbarus hic ergo sum, quia non intellegor ulli.

Literatur

1 Guttormsen S, Perruchoud AP. Pionierarbeit für die Abschlussprüfung Medizin. Schweiz Ärztezeitung. 2015;96(50-51):1846-8.

Bildnachweis

(c) Megaflopp | Dreamstime.com 